\title{
Long-Term Outcomes after Dialysis-Requiring Acute Kidney Injury
}

\section{Vin-Cent Wu, ${ }^{1}$ Chih-Chung Shiao, ${ }^{2}$ Chia-Hsuin Chang, ${ }^{1}$ Tao-Min Huang, ${ }^{3}$ Chun-Fu Lai, ${ }^{1}$ Meng-Chun Lin, ${ }^{1}$ Wen-Chih Chiang, ${ }^{1}$ Tzong-Shinn Chu, ${ }^{1}$ Kwan-Dun Wu, ${ }^{1}$ Wen-Je Ko, ${ }^{4}$ Cheng-Yi Wang, ${ }^{5}$ Shuo-Meng Wang, ${ }^{6}$ and Likwang Chen ${ }^{7}$}

\author{
${ }^{1}$ Division of Nephrology, Department of Internal Medicine, National Taiwan University Hospital, 7 Chung-Shan South Road, \\ Zhong-Zheng District, Taipei 100, Taiwan \\ ${ }^{2}$ Division of Nephrology, Department of Internal Medicine, Saint Mary's Hospital, Saint Mary's Medicine, Nursing, \\ and Management College, Luodong, Yilan 265, Taiwan \\ ${ }^{3}$ Division of Nephrology, Department of Internal Medicine, Yun-Lin Branch, National Taiwan University Hospital, \\ Douliou City 640, Taiwan \\ ${ }^{4}$ Department of Traumatology and Surgery, National Taiwan University Hospital, 7 Chung-Shan South Road, \\ Zhong-Zheng District, Taipei 100, Taiwan \\ ${ }^{5}$ Department of Internal Medicine, Medical Research Center, Cardinal Tien Hospital, School of Medicine, \\ Fu Jen Catholic University, No. 362, Zhongzheng Road, Xindian District, New Taipei City 23148, Taiwan \\ ${ }^{6}$ Department of Urology, National Taiwan University Hospital, 7 Chung-Shan South Road, Zhong-Zheng District, \\ Taipei 100, Taiwan \\ ${ }^{7}$ Institute of Population Health Sciences, National Health Research Institutes, No. 35, Keyan Road, Zhunan 350, Taiwan
}

Correspondence should be addressed to Cheng-Yi Wang; cywang@mospital.com and Shuo-Meng Wang; turo62@yahoo.com.tw Received 5 February 2014; Revised 10 May 2014; Accepted 9 June 2014; Published 12 August 2014

Academic Editor: Raul Lombardi

Copyright (C) 2014 Vin-Cent Wu et al. This is an open access article distributed under the Creative Commons Attribution License, which permits unrestricted use, distribution, and reproduction in any medium, provided the original work is properly cited.

AKI-dialysis patients had a higher incidence of long-term ESRD and mortality than the patients without AKI. The patients who recovered from dialysis were associated with a lower incidence of long-term ESRD and mortality than in the patients who still required dialysis.

\section{Introduction}

The incidence of acute kidney injury (AKI) in hospitalized patients is increasing [1] and is associated with increased in-hospital and posthospitalization resource utilization [2]. Patient survival from an episode of AKI has been improved by advances in critical care medicine and dialysis technology, and therefore an increasing number of hospitalized patients are being discharged alive after temporary AKI [3]. Patients who survive AKI have been reported to be at a greater risk for end-stage renal disease (ESRD) than patients without AKI $[4,5]$, and pediatric patients without preexisting kidney disease have been reported to be at a higher risk of chronic kidney disease after AKI [6]. However, the results of longterm outcomes of patients recovering from in-hospital AKI necessitating dialysis (AKI-dialysis) have been inconsistent [5, 7-9]. Although renal recovery from AKI is associated with better renal outcomes and patient survival [10], no differences in long-term survival between those with kidney function recovery after AKI and those without AKI were observed in two population-based cohorts [11, 12]. However, in postoperative patients [13-15] and geriatric patients [16], temporary worsening of kidney function has been reported with a higher long-term mortality rate compared with nonAKI patients.

The cohorts in previous reports have mostly focused on preexisting normal or near normal kidney function $[9$, 17-19] or all patients with chronic kidney disease (CKD) [8]. We hypothesized that hospitalized patients surviving 
with temporary dialysis would have poorer long-term allcause mortality than patients without AKI in a communitybased cohort of patients with and without CKD. We also compared the long-term outcomes of hospital survivors who still required dialysis. This study was conducted using 1 million beneficiaries randomly sampled from the year 2000 by the Taiwan National Health Research Institute (NHRI) and further validated by analysis of a prospectively constructed AKI database.

\section{Patients and Methods}

2.1. Study Population. The Taiwan National Health Insurance (NHI) program is a nationwide insurance program that covers outpatient visits, hospital admissions, prescriptions, intervention procedures, and disease profiles for over $99 \%$ of the population in Taiwan (23.12 million in 2009). It is one of the largest and most comprehensive databases in the world and has been used extensively in various studies on prescription use, diagnoses, and hospitalizations [2022]. In cooperation with the Bureau of NHI, the National Health Research Institute (NHRI) of Taiwan randomly samples a representative database of 1,000,000 subjects from all enrollees in the NHI program using a systematic sampling method for research purposes in the form of the NHRI database (NHRID). There are no statistically significant differences in age, gender, and health-care costs between the sample group and all enrollees according to the NHRI. The NHRID contains all claims data for these individuals from January 1999 to December 2008 and offers a good opportunity to explore the outcomes of AKI-dialysis. Because the identification numbers of all subjects in the NHRID are encrypted to protect privacy, this study was exempt from full review by the Institutional Review Board.

2.2. Identification of Cases and Controls. The study group consisted of those aged $\geq 18$ years with a first diagnosis of AKIdialysis according to International Classification of Disease, 9th revision, Clinical Modification (ICD-9-CM) code and procedure codes (including the Taiwan Classification of Procedures, supplementary tables). A control cohort without AKI or dialysis before and during the index hospitalization was selected for comparison (non-AKI group), matched for age, sex, diabetes mellitus (DM), and mechanical ventilation (MV) support with the study group. The index hospitalization date of the controls was limited to be within the same year as that of their matched cases.

We used a one-year period immediately prior to the index hospitalization to identify preadmission AKI and dialysis. Patients with preadmission AKI or ESRD and those who had undergone kidney transplantation were excluded. Figure 1 shows the patient selection flow chart. Patients with an arteriovascular fistula or implantation of a peritoneal-dialysis tube were also excluded. The AKI-dialysis patients who survived for more than 90 days after discharge from the index hospitalization and who were not readmitted to hospital were divided into two groups according to whether or not they recovered from AKI-dialysis (dialysis withdrawal and nonwithdrawal subgroups). We further defined advanced CKD as patients with a creatinine level of more than $6 \mathrm{mg} / \mathrm{dl}$ with prescriptions of concomitant erythropoiesis-stimulating agents [23]. Further, as previously reported [24], we used a selection period of 90 days to define ESRD because all patients receiving dialysis for more than 90 days in Taiwan can apply for NHI for catastrophic illness registration cards. The outcomes of this study were long-term all-cause mortality and ESRD after hospital discharge.

2.3. Research Variables. The demographic and clinical characteristics of the study subjects at their index hospitalization were recorded. The parameters included age, sex, year of admission, hospital characteristics, prevalence of selected comorbid conditions, Charlson comorbidity index [25], organ dysfunction developing during the index hospitalization, the categories of major operations, resource usage including hemodialysis and MV support, ICU admission, and outcomes. To determine preexisting comorbidities, we used a relatively strict criterion: at least one inpatient admission or at least three outpatient visits to treat a certain disease during the year prior to the index hospitalization. Moreover, medications including angiotensin-converting-enzyme inhibitors (ACEIs), angiotensin II receptor blockers (ARBs), statins, nonsteroidal anti-inflammatory agents (NSAIDs), diuretics, and aspirin, which are thought to influence kidney recovery [26] and were used during the 90 days after the index hospitalization, were also analyzed.

We also make an effort to examine how fluid imbalance at the initiation of dialysis could affect long-term all-cause mortality. Ideally, it would be more informative to construct a research variable to reflect the level of fluid overload based on the percentage of body weight gained. However, the NHI database does not contain data on patients' body weight. Alternatively, we constructed a proxy indicator of fluid imbalance based on the amount of medication used to control oliguria and body weight gain. We generated a variable to show whether a patient used diuretics at a level higher than 2.25 defined daily dose (DDD) at the initiation of dialysis. Each patient's exposure to diuretics (belonging to the anatomical, therapeutic, and chemical (ATC) class C03CA) was measured on the basis of the cumulative dose and expressed as the DDD according to the definition of the World Health Organization [27]. The DDD level of 2.25 was chosen as the dose equivalent of furosemide stress test $(1.5 \mathrm{mg} / \mathrm{kg})$ from a standard $60 \mathrm{~kg}$ patient [28]. This dosage signals a severe level of fluid imbalance.

2.4. Statistical Analysis. Continuous variables are described as mean \pm standard deviation (SD), and discrete variables are presented as counts or percentages. All data were analyzed using $\mathrm{R}$ software version 2.8.1 (Free Software Foundation, Inc., Boston, MA, USA). A two-sided $P$ value of less than 0.05 was considered to be statistically significant. Cox proportional hazard regression and propensity score analyses were conducted separately within each stratum to evaluate the risk of outcomes after adjustments for all variables in Table 2 and propensity score. For the outcome measurements, 


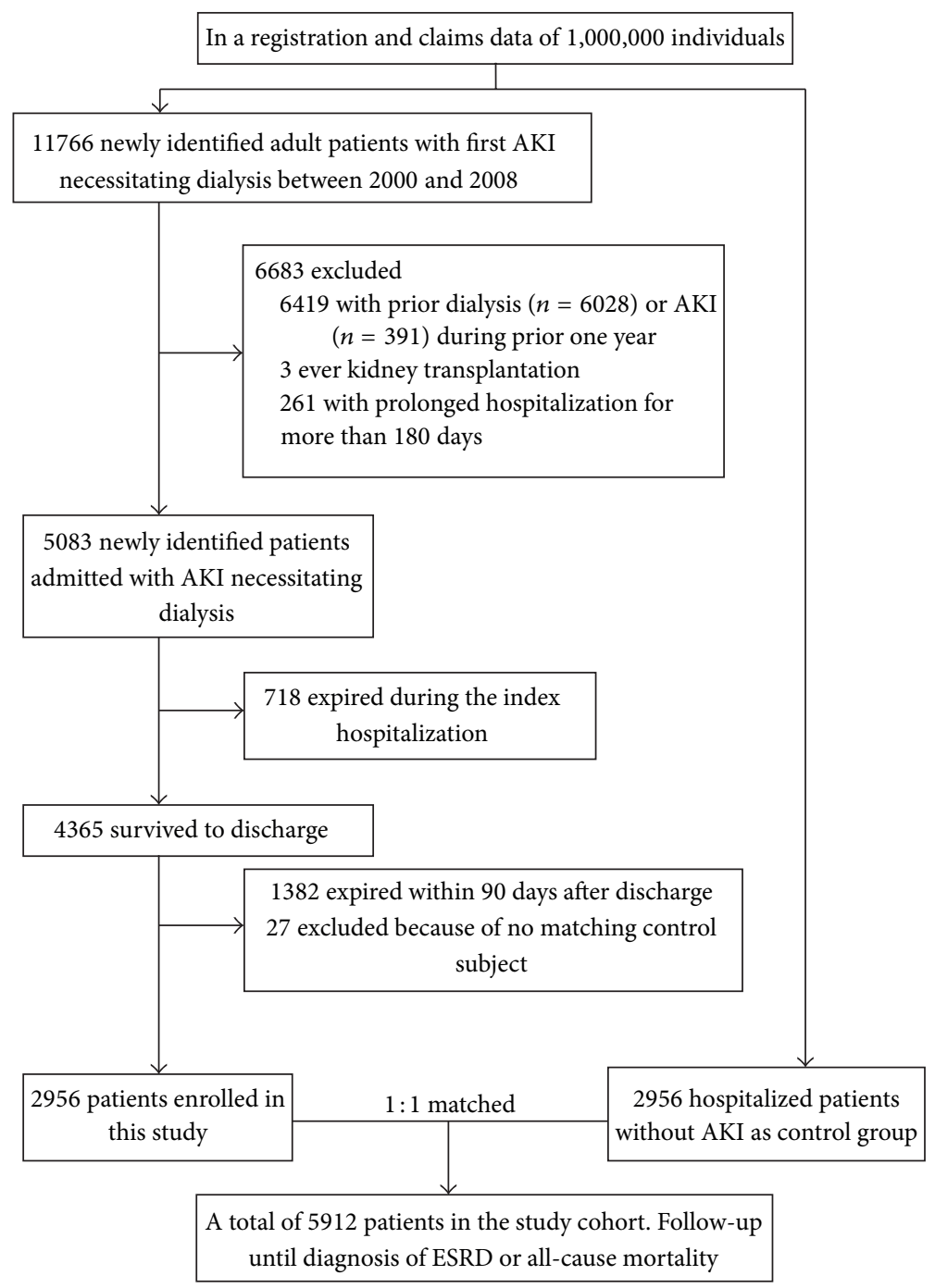

FIGURE 1: Flow diagram of the study population (AKI, acute kidney injury; DM, diabetes mellitus; ESRD, end-stage renal disease; MV, mechanical ventilation).

an individual was censored at death or at the end of the measured period.

We calculated propensity scores in an attempt to make an unbiased estimate of the confounders predicting dialysis at the 90th day after discharge, as a binary dependent variable, under a set of covariates (see Supplementary Table 1 in Supplementary Materials available online at http://dx.doi.org/ $10.1155 / 2014 / 365186)$. The presence of comorbidities was added into a nonparsimonious multivariate logistic regression model to predict dialysis at the 90th day after hospital discharge. The predicted probability derived from the logistic equation was used as the propensity score for each individual.

Due to the strong correlation between CKD, advanced CKD, ESRD, and mortality [29], we further used a Cox proportional hazards model with time-varying covariates to evaluate the impact of subsequent ESRD, advanced CKD, and CKD after discharge on the risk of mortality, assuming that changes in CKD, advanced CKD, or ESRD status could appear at a subsequent time point.

\section{Validation}

3.1. Propensity Matching Method for Sensitivity. The propensity score matching method was applied in the dialysis withdrawal and nonwithdrawal subgroups or non-AKI patients to reduce the effect of selection bias in the cohorts as in our previous reports $[30,31]$. The subjects who did not have a suitable match within the acceptable rank range were excluded from further analysis. The models were applied to the non-AKI and nonwithdrawal groups, with the dialysis withdrawal subgroup as the reference. The patients in the dialysis withdrawal subgroup were then matched 1:1 separately with the nonwithdrawal subgroup and non-AKI group according to their specific propensity scores using the greedy matching technique as in our previous report [32].

3.2. Validation of Data Collection. The main outcome of allcause mortality and the selection criteria to identify patients 
with AKI-dialysis were validated by analysis of prospectively collected data from the National Taiwan University Hospital Study Group on Acute Renal Failure (NSARF). This critical care database was constructed prospectively for outcome assessment between January 2002 and January 2008 in a single medical center (National Taiwan University Hospital in Taipei, Taiwan) and its three branch hospitals in different cities [15,33-37] with complete information on serum creatinine (measured by following a standardized protocol). The contents of this database were used for reimbursements and are similar to those of the NHI inpatient claims files.

\section{Results}

\subsection{Demographic Characteristics of the Patients}

4.1.1. Patients with or without AKI-Dialysis. Of 5083 hospitalized patients with de novo AKI-dialysis, 718 (14.1\%) died during the index hospitalization, and a total of $41.3 \%$ patients died from hospitalization to 90 days after discharge (most of whom were discharged with do not resuscitate orders [38]) (Figure 1). After excluding 27 patients for whom a matching control subject could not be found, 2956 AKI-dialysis patients were enrolled with 2956 non-AKI matched inpatients (men, $50.4 \%$; mean age, $62.0 \pm 14.8$ years). In the whole cohort, the average age was 62.0 years and the Charlson score before admission was $2.62 \pm 2.25$. A total of 5912 patients who survived for more than 90 days after hospital discharge were included for analysis, of whom 53.8\% had DM and $17.5 \%$ needed MV support (Table 1).

The patients in the AKI-dialysis group had a higher Charlson score, with more preexisting comorbidities $(P<$ 0.001 ) and more comorbidities during the index hospitalization than the non-AKI group. More patients in the nonAKI group had a history of surgery at admission than in the AKI-dialysis group, however, and the non-AKI patients were more likely to undergo major surgery during the index hospitalization. More AKI-dialysis patients took medications including ACEIs, ARBs, statins, NSAIDs, and diuretics after hospital discharge.

4.1.2. Patients with or without Withdrawal from AKI-Dialysis. Of the AKI-dialysis patients who survived to 90 days after hospital discharge, $685(23.2 \%)$ were weaned from acute dialysis (Table 1). These patients had lower Charlson scores and a lower rate of comorbidities (myocardial infarction (MI), dementia, milder liver disease, and DM with microvascular disease) than the nonwithdrawal group. However, the dialysis withdrawal patients received more major operations, MV support, and intensive care unit (ICU) admission, with a higher rate of cardiovascular and respiratory organ failure during the index hospitalization than those in the nondialysis withdrawal group. As expected, the ratio of baseline CKD was lower in the dialysis withdrawal group than in the nonwithdrawal group.

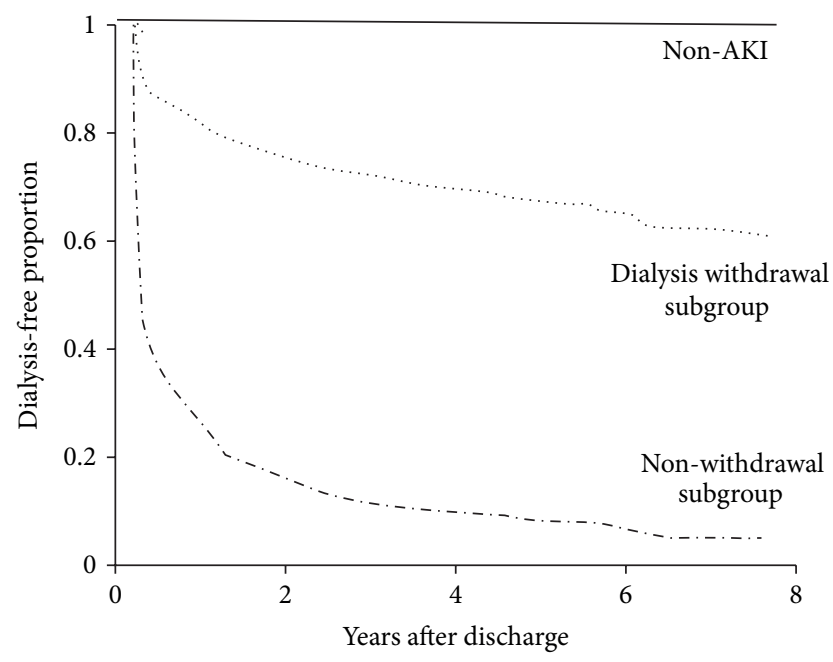

FIGURE 2: Cox proportional hazard model for long-term dialysis events of the patients alive at hospital discharge, stratified by kidney functional status after discharge (AKI, acute kidney injury).

\subsection{Outcome Measurements}

4.2.1. Propensity Score Evaluation. The risk factors predicting the need for dialysis within 90 days after hospital discharge as components of propensity score are listed in Supplementary Table S1. The propensity score for predicting the need for dialysis within 90 days after hospital discharge in all study groups had a high discriminatory power (estimated area under receiver operating characteristic curves (eAUCROC) $=0.895)$ and it fitted well with the observed binary data (adjusted generalized $R^{2}=0.588$ ).

4.2.2. Long-Term ESRD. The incidence of ESRD was 6.8 per 100 person-years among the dialysis withdrawal subgroup after a median follow-up period of 2.96 years (interquartile range (IQR)) (0.49-4.83 years). The non-AKI group had a significantly lower incidence of long-term dialysis (hazard ratio (HR), $0.05,95 \%$ confidence interval (CI), $0.02-0.12, P<$ 0.001 ), and the nonwithdrawal subgroup had worse outcomes (HR, 10.38, 95\% CI $8.02-13.42, P<0.001$ ) of long-term dialysis compared with the dialysis withdrawal subgroup as the reference (Figure 2). In addition, preadmission advanced CKD (HR, 1.23, 95\% CI, 1.09-1.38, $P=0.001$ ), intermittent hemodialysis use during the index hospitalization (HR, 2.36, 95\% CI, 1.21-4.58, $P=0.012$ ), and postdischarge ACEI/ARB use (HR, $0.82,95 \% \mathrm{CI}, 0.73-0.91, P=0.001$ ) were associated with long-term dialysis.

4.2.3. Long-Term All-Cause Mortality. The survivors were younger and had fewer comorbidities than the nonsurvivors (Table 2). During the index hospitalization, the survivors also had a lower rate of respiratory disease. The surviving patients received fewer cardiothoracic surgeries, used less MV support, had fewer ICU admissions, and used lower amounts of statins, aspirin, and diuretics than the nonsurvivors. Consistent with previous results, our findings showed 


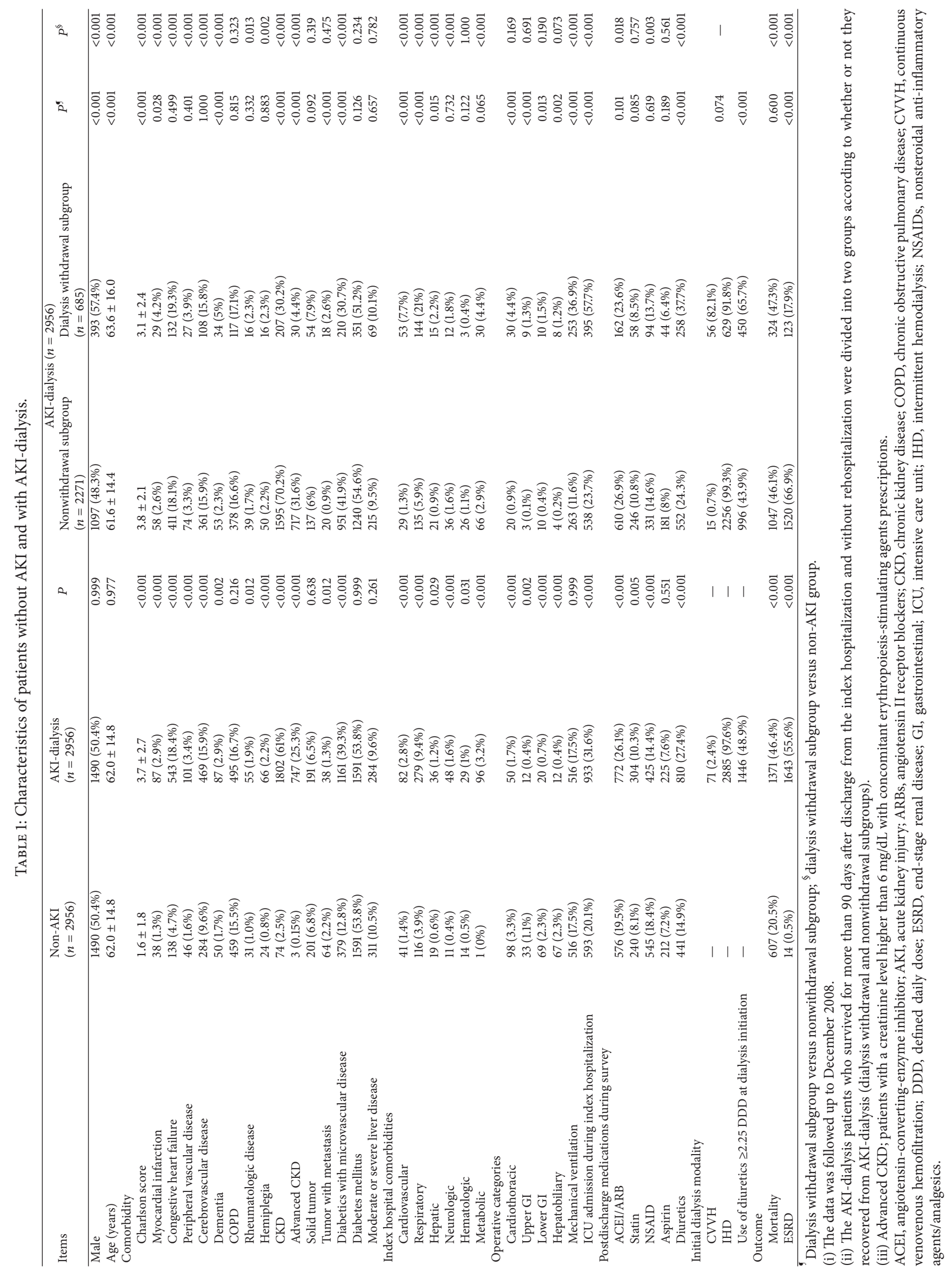


TABLE 2: Characteristics of patients with or without long-term survival.

\begin{tabular}{|c|c|c|c|}
\hline & Survival $(n=3934)$ & Nonsurvival $(n=1978)$ & $P$ \\
\hline Male & $1946(49.5 \%)$ & $1034(52.3 \%)$ & 0.044 \\
\hline Age (years) & $58.9 \pm 14.9$ & $68.1 \pm 12.5$ & $<0.001$ \\
\hline \multicolumn{4}{|l|}{ Comorbidity } \\
\hline Charlson score & $2.1 \pm 2.0$ & $3.7 \pm 2.3$ & $<0.001$ \\
\hline Myocardial infarction & $62(1.6 \%)$ & $63(3.2 \%)$ & $<0.001$ \\
\hline Congestive heart failure & $283(7.2 \%)$ & $398(20.1 \%)$ & $<0.001$ \\
\hline Peripheral vascular disease & $70(1.8 \%)$ & $77(3.9 \%)$ & $<0.001$ \\
\hline Cerebrovascular disease & $353(9.0 \%)$ & $400(20.2 \%)$ & $<0.001$ \\
\hline Dementia & $52(1.3 \%)$ & $85(4.3 \%)$ & $<0.001$ \\
\hline COPD & $497(12.6 \%)$ & $457(23.1 \%)$ & $<0.001$ \\
\hline Rheumatologic disease & $55(1.4 \%)$ & $31(1.6 \%)$ & 0.645 \\
\hline Hemiplegia & $30(0.8 \%)$ & $60(3.0 \%)$ & $<0.001$ \\
\hline Chronic kidney disease & $1008(25.6 \%)$ & $868(43.9 \%)$ & $<0.001$ \\
\hline Solid tumor & $212(5.4 \%)$ & $180(9.1 \%)$ & $<0.001$ \\
\hline Tumor with metastasis & $38(0.9 \%)$ & $64(3.2 \%)$ & $<0.001$ \\
\hline Diabetics with microvascular disease & $819(20.8 \%)$ & $721(36.5 \%)$ & $<0.001$ \\
\hline Diabetes mellitus & $1972(50.1 \%)$ & $1210(61.2 \%)$ & $<0.001$ \\
\hline Moderate or severe liver disease & $355(9.02 \%)$ & $240(12.1 \%)$ & $<0.001$ \\
\hline \multicolumn{4}{|l|}{ Index hospital comorbidity } \\
\hline Cardiovascular & $73(1.9 \%)$ & $50(2.5 \%)$ & 0.100 \\
\hline Respiratory & $200(5.1 \%)$ & $195(9.9 \%)$ & $<0.001$ \\
\hline Hepatic & $35(0.9 \%)$ & $20(1.01 \%)$ & 0.668 \\
\hline Neurologic & $34(0.9 \%)$ & $25(1.3 \%)$ & 0.165 \\
\hline Hematologic & $25(0.6 \%)$ & $18(0.9 \%)$ & 0.258 \\
\hline Metabolic & $63(1.6 \%)$ & $34(1.7 \%)$ & 0.745 \\
\hline \multicolumn{4}{|l|}{ Initial dialysis modality } \\
\hline CVVH & $48(1.2 \%)$ & $23(1.2 \%)$ & \multirow{2}{*}{$<0.001$} \\
\hline IHD & $1537(39.1 \%)$ & $1348(68.1 \%)$ & \\
\hline Use of diuretics $\geq 2.25 \mathrm{DDD}$ at dialysis initiation & $999(25.4 \%)$ & $849(42.9 \%)$ & $<0.001$ \\
\hline \multicolumn{4}{|l|}{ Operative categories } \\
\hline Cardiothoracic & $113(2.9 \%)$ & $35(1.8 \%)$ & 0.010 \\
\hline Upper GI & $26(0.7 \%)$ & $19(0.96 \%)$ & 0.209 \\
\hline Lower GI & $54(1.4 \%)$ & $35(1.8 \%)$ & 0.258 \\
\hline Hepatobiliary & $59(1.5 \%)$ & $20(1.01 \%)$ & 0.149 \\
\hline Mechanical ventilation & $633(16.1 \%)$ & $399(20.2 \%)$ & $<0.001$ \\
\hline ICU admission during index hospitalization & $886(22.5 \%)$ & $640(32.4 \%)$ & $<0.001$ \\
\hline \multicolumn{4}{|l|}{ Postdischarge medications during survey periods } \\
\hline ACEI/ARB & $875(22.2 \%)$ & $473(23.9 \%)$ & 0.149 \\
\hline Statin & $403(10.2 \%)$ & $141(7.1 \%)$ & $<0.001$ \\
\hline NSAID & $651(16.6 \%)$ & $319(16.1 \%)$ & 0.710 \\
\hline Aspirin & $271(6.9 \%)$ & $166(8.4 \%)$ & 0.040 \\
\hline Diuretics & $654(16.6 \%)$ & $597(30.2 \%)$ & $<0.001$ \\
\hline \multicolumn{4}{|l|}{ Study categories } \\
\hline Non-AKI & $2349(59.7 \%)$ & $607(30.7 \%)$ & \multirow{3}{*}{$<0.001$} \\
\hline Dialysis withdrawal subgroup & $361(9.2 \%)$ & $324(16.4 \%)$ & \\
\hline Nonwithdrawal subgroup & $1224(31.1 \%)$ & $1047(52.9 \%)$ & \\
\hline ESRD & $985(25.0 \%)$ & $672(34.0 \%)$ & $<0.001$ \\
\hline
\end{tabular}

ACEI, angiotensin-converting-enzyme inhibitor; AKI, acute kidney injury; ARBs, angiotensin II receptor blockers; COPD, chronic obstructive pulmonary disease; DDD, defined daily dose; ESRD, end-stage renal disease; GI, gastrointestinal; ICU, intensive care unit; NSAIDs, nonsteroidal anti-inflammatory agents/analgesics. 


\begin{tabular}{l}
\multicolumn{1}{c}{ Number of events at risk } \\
\multicolumn{1}{c}{ Dialysis } \\
withdrawal
\end{tabular}

FIGURE 3: Hazard ratios and 95\% confidence intervals for overall survival after adjusting for patient characteristics between the dialysis-withdrawal subgroup and the AKI-dialysis and non-AKI groups (AKI, acute kidney injury; CI, confidence interval; COPD, chronic obstructive pulmonary disease; HR, hazard ratio; ICU, intensive care unit).

a lower incidence of dialysis, AKI, and ESRD, but a high incidence of kidney recovery among the surviving patients (Table 2).

Even among the dialysis withdrawal patients, the mortality rate was 14.4 per 100 person-years after a mean followup period of 3.29 years. The all-cause mortality rate of 4.45 per 100 person-years in the matched controls in our cohort was consistent with previous reports $[5,39]$. After a mean follow-up period of $4.1 \pm 2.6$ years, the non-AKI group had a lower risk (HR, $0.65,95 \%$ CI, $0.43-0.83, P<$ 0.001 ), whereas the nonwithdrawal subgroup had a higher risk (HR, 1.63, 95\% CI, 1.39-1.92, $P<0.001$ ) of long-term all-cause mortality compared with the withdrawal group. The risk was independent from use of diuretics $\geq 2.25 \mathrm{DDD}$ at dialysis initiation (HR, 1.15, 95\% CI, 1.03-1.28, $P=$ 0.011 ) and development of subsequent ESRD (HR, 1.70, 95\% CI, 1.47-1.98, $P<0.001)$ and CKD $(P=0.456)$ after discharge (Table 3). The subgroup analysis was consistent with our main finding that the non-AKI group had a survival advantage compared with the dialysis withdrawal subgroup (Figure 3).

\subsection{Sensitivity Test}

4.3.1. Propensity Matching Method. After careful matching, there were 231 dialysis withdrawal patients and 231 non-AKI patients. Supplementary Table 2(a) shows the demographic data of the matched cohort. Consistent with our previous findings, the results showed that the non-AKI patients had a lower long-term mortality rate (HR, $0.62 ; 95 \% \mathrm{CI}, 0.44-0.88$; $P=0.007$ ) and long-term ESRD (HR, 0.04; 95\% CI, 0.01-0.14; $P<0.001)$ than the dialysis withdrawal subgroup.

We also performed one-to-one matching between the dialysis withdrawal $(n=543)$ and $(n=543)$ nonwithdrawal groups according to each patient's propensity score (Supplementary Table 2(b)). With regard to the demographic data, the nondialysis withdrawal subgroup had a higher long-term mortality rate (HR, $1.25 ; 95 \% \mathrm{CI}, 1.06-1.48 ; P=0.008)$ and ESRD (HR, 9.45; 95\% CI, 6.57-13.59; $P<0.001$ ) than the dialysis withdrawal subgroup.

4.4. Validation Using NSARF Data (Supplementary Table 3). We validated our main findings using prospective critical care data from the NSARF. Among 234 AKI-dialysis patients, 
TABLE 3: Risk of long-term mortality stratified by dialysis status after index hospital discharge by Cox proportional hazard model with timevarying covariates.

\begin{tabular}{|c|c|c|c|c|}
\hline & HR & Lower 95\% CI & Upper 95\% CI & $P$ \\
\hline Age (per year) & 1.04 & 1.04 & 1.05 & $<0.001$ \\
\hline Charlson score & 1.17 & 1.13 & 1.20 & $<0.001$ \\
\hline Male & 1.28 & 1.17 & 1.40 & $<0.001$ \\
\hline \multicolumn{5}{|l|}{ Groups } \\
\hline Nonwithdrawal versus dialysis withdrawal subgroup & 1.63 & 1.39 & 1.91 & $<0.001$ \\
\hline Non-AKI versus dialysis withdrawal subgroup & 0.65 & 0.43 & 0.83 & $<0.001$ \\
\hline Dementia & 1.34 & 1.07 & 1.69 & 0.012 \\
\hline Severe liver disease & 1.88 & 1.39 & 2.56 & 0.012 \\
\hline \multicolumn{5}{|l|}{ During index hospitalization } \\
\hline Respiratory failure during index hospitalization & 1.24 & 1.05 & 1.47 & $<0.001$ \\
\hline Lower GI surgery & 1.63 & 1.15 & 2.29 & 0.005 \\
\hline ICU admission during index hospitalization & 1.22 & 1.09 & 1.37 & $<0.001$ \\
\hline Use of diuretics $\geq 2.25 \mathrm{DDD}$ at dialysis initiation & 1.15 & 1.03 & 1.28 & 0.011 \\
\hline Statin use after discharge & 0.77 & 0.65 & 0.92 & 0.003 \\
\hline Diuretics use after discharge & 1.28 & 1.15 & 1.41 & $<0.001$ \\
\hline Time-varying ESRD event & 1.70 & 1.47 & 1.98 & $<0.001$ \\
\hline
\end{tabular}

Adjusted generalized $R^{2}=0.279$; concordance index $=0.83$.

AKI, acute kidney injury; CI, confidence interval; DDD, defined daily dose; ESRD, end-stage renal disease; GI, gastrointestinal; HR, hazard ratio.

$180(76.9 \%)$ recovered from dialysis within 90 days after discharge from index admission. In the NSARF cohort, 8788 non-dialysis patients who survival to 90 days after hospital discharge were enrolled as the controls. The baseline CKD rates, defined as patients with a baseline estimated glomerular filtration rate $\leq 60 \mathrm{~mL} / \mathrm{min} / 1.73 \mathrm{~m}^{2}$, were $6.9 \%$, $30.7 \%$, and $63.0 \%$ among the non-AKI, dialysis withdrawal, and nonwithdrawal groups, respectively. The AKI-dialysis patients had an average Charlson score of $3 \pm 3.4$ and an average acute physiology and chronic health evaluation II score of $18.4 \pm 8.7$.

Consistent with our previous findings, the results obtained using the Cox proportional hazard model showed that the nonwithdrawal subgroup had a significantly higher long-term mortality rate during the follow-up period ( $\mathrm{HR}$, $1.64 ; 95 \% \mathrm{CI}, 1.05-2.56 ; P=0.031)$. In addition, the non-AKI group had better survival (HR, 0.69; 95\% CI, 0.54-0.87; $P=0.002)$ than the dialysis withdrawal subgroup after a median (IQR) follow-up of 3.9 years (2.38-5.65 years).

\section{Discussion}

Patients with dialysis-requiring AKI, even temporary dialysis, had a higher long-term mortality rate than those with neither AKI nor dialysis in this large, community-based cohort of patients with and without CKD. The results using NHI data (retrospectively collected) and in NSARF data (prospectively collected) were similar. These findings are important from the perspective of a clinician caring for an individual with temporary dialysis-requiring AKI.

5.1. Dialysis Withdrawal and Long-Term ESRD. AKI is accompanied by extrarenal organ system failure in most patients [40]. Although there was a high mortality rate in the AKI-dialysis patients, nearly one-fifth of the surviving patients had kidney function recovery attesting to the remarkable ability of the kidneys to repair and regenerate even after severe dialysis-requiring injury. Our findings also highlight the magnitude of the problem of AKI as a cause of ESRD. According to our results, the estimated annual incidence of ESRD due to temporary dialysis was 6.8 per 100 person-years. In particular, we provided an important quantitative estimate; that is, even in survival during hospitalization and recovery of sufficient kidney function to stop dialysis, a high incidence of chronic dialysis was still required.

Severe ischemic injury results in a permanent alteration of renal capillary density, contributing to a urinary concentrating defect and a predisposition toward the development of renal fibrosis [41]. Furthermore, damage of residual kidney structure, which has been identified after AKI in animal models, includes tubular atrophy and dilation, interstitial fibrosis, and a reduction in peritubular capillary density [42, 43]. These findings suggest that AKI is associated with an increased risk of ESRD [16].

Taken together, our results show a graded relationship between AKI and ESRD, with a greater risk associated with nonrecovery from dialysis. AKI is therefore a nonnegligible cause of ESRD; however the reason why this is the case is more difficult to answer. Further research is needed to elucidate whether AKI accelerates the normal age-related decline in glomerular filtration rate [44] or whether it is a marker for other factors that are causally related to the development of kidney failure.

The impact of the initial renal replacement modality in critically ill patients with AKI on recovery of kidney function is an area of renewed interest [45]. As per our previous report, initial renal support with continuous RRT showed 
great advantage to dialysis independence than intermittent RRT [24].

5.2. Long-Term All-Cause Mortality. Our long-term outcomes do not concur with two previous studies that showed no increased mortality associated with temporary acute dialysis $[11,12]$. Wald et al. reported that a prior history of AKI and dialysis was not independently associated with long-term mortality in a population-based cohort after excluding $7 \%$ of the high-risk patients [12]. The discrepancy may be attributed to the higher Charlson comorbidity score and the higher rates of DM and CKD in our study cohort from national claims data, which resulted in a higher annual mortality rate in our dialysis withdrawal subgroup (14.4\% versus $10.1 \%$ in Wald et al.s study [12]). Given the extraordinarily high rates of ESRD and mortality observed in the temporary dialysis patients, the complex interconnection between them, and the increasing incidence of both, kidney disease prevention and treatment should be a major public health priority. Furthermore, the findings of the current study also have important regional implications. Appropriate management of CKD, AKI, and ESRD is important in Taiwan not only because of the high prevalence of CKD, but also because Taiwan has the highest prevalence of ESRD in the world [46]. Consistent with our findings, use of diuretics $\geq 2.25$ DDD at dialysis initiation predicted a worse outcome in the patients with AKI [47]. Whether the fluid imbalance was the result of more severe renal failure or whether it contributed to its cause requires further clinical trials to elucidate.

In patients with normal renal function $(>90 \mathrm{~mL} /$ $\min / 1.73 \mathrm{~m}^{2}$ ) prior to the renal insults who survive the precipitating cause of $\mathrm{AKI}$, the overwhelming majority have been reported to recover sufficient renal function (inpatient death rate, 53\%; withdrawal from dialysis, 100\%) [18]. The RENAL study reported a high initial death rate $(44.6 \%)$ with a low rate of those requiring maintenance dialysis (5.4\% of those at day 90 after discharge). This is also supported by the fact that the enrollees in RENAL study had mean creatinine level of around $1.5-1.76 \mathrm{mg} / \mathrm{dL}$ before randomization. In patients with preexisting normal or near normal kidney function $\left(>45 \mathrm{~mL} / \mathrm{min} / 1.73 \mathrm{~m}^{2}\right)$ the inpatient death rate was $41 \%$ and withdrawal from dialysisrequiring $\mathrm{AKI}$ was 84\% [9]. However, in patients with advanced CKD (average $15-29 \mathrm{~mL} / \mathrm{min} / 1.73 \mathrm{~m}^{2}$ ), an episode of superimposed dialysis-requiring AKI was associated with a low rate of inpatient death $(28 \%)$ and a very low likelihood of recovering renal function $(37.0 \%)$ [8].

Consistent with the results, the inpatient death rate of our cohort was $15.4 \%$, and the recovery rate was low (23.2\%). Thus most of the patients with dialysis-requiring AKI in this study had stage $-3 / 4$ or worse CKD, and $25.3 \%$ had advanced CKD. Therefore, most of our study group had advanced CKD superimposed with dialysis-requiring AKI, and this is consistent with our Cox analysis in the fact that baseline advanced CKD was a risk for long-term dialysis.

Accordingly, if AKI-dialysis leads to a persistent loss of renal function, then the resulting renal function impairment would account for the increased mortality [48]. Our study findings highlight that, even after adjusting for subsequent CKD or ESRD after hospital discharge, the effect of AKI is still significant. In patients after aortic surgery, the temporary worsening of renal function led to a poor long-term mortality rate compared to non-AKI patients with more preexisting comorbidities [13]. Higher comorbidities in temporary dialysis patients will result in more cardiovascular events compared with patients without AKI, and this may be the reason why AKI is a cause of mortality [49].

AKI patients with higher frailty had a higher mortality rate as expected, and the corresponding analysis would therefore lead to higher adjusted HRs for death when these patients were compared with the non-AKI patients, especially among inpatients after AKI-dialysis. This phenomenon underlies the significance of checking inherent frailty among research subjects in outcome comparisons between AKI and nonAKI. Nonetheless, the dialysis withdrawal subgroup still had poorer outcomes than those without AKI or dialysis in all scenarios. It has been reported that only $8.5 \%$ of AKI patients are referred to a nephrologist after discharge [50]. However, early postdischarge followup with a nephrologist in survivors of dialysis-requiring AKI is necessary and has been associated with a lower risk of death [51].

5.3. Study Limitations. Despite careful propensity score analysis, we cannot exclude the possibility of residual confounding by changes in the urine amount, serum creatinine level, and body weight gain during the index hospitalization. Nonetheless, we used procedure codes to define AKIdialysis and focused on the patients who were weaned from temporary dialysis in the NHI reimbursement system which has a high accuracy. Further studies on dialysissparing AKI on patient outcomes are necessary. Second, we defined AKI as occurring from any cause and thus we were unable to detect whether the risk of progression to an adverse outcome differed among different etiologies of AKI. The major shortcoming of using administrative data as the primary basis for matching is misclassification of exposure status, specifically in this study for the presence or absence of CKD. However, the excellent performance of administrative data sets stratified by ICD-9-CM codes for AKI with dialysis has been verified to be suited for research purposes with both sensitivity and specificity of more than 90\% [52].

\section{Conclusions}

Our results reinforce the view that dialysis-requiring AKI seems to independently increase all-cause mortality, even after adjusting for preexisting and subsequent CKD or ESRD. AKI itself may require specialized care to rigorously avoid potentially nephrotoxic factors, despite recovering from dialysis and AKI, after discharge that may hasten progression to long-term ESRD, and then all-cause mortality.

\section{Abbreviations}

ACEI: Angiotensin-converting-enzyme inhibitor

AKI: Acute kidney injury 
ARBs: Angiotensin II receptor blockers

COPD: Chronic obstructive pulmonary disease

ESRD: End-stage renal disease

GI: Gastrointestinal

ICU: Intensive care unit

NSAIDs: Nonsteroidal anti-inflammatory agents/analgesics.

\section{Conflict of Interests}

No conflict of interests is reported.

\section{Authors' Contribution}

Cheng-Yi Wang and Shuo-Meng Wang contributed equally to this work.

\section{Acknowledgments}

The authors would like to thank the staff of the Second, Seventh and Eighth Core Lab of the Department of Medical Research in National Taiwan University Hospital for technical assistance. The authors express their sincere gratitude to all participants of the NSARF. This study was supported by Grants: NSC-102-2314-B-002-140-MY2, NSC 101-2314-B002-132-MY3, NSC 101-2314-B-002-085-MY3, NSC100-2314B-002-119, NSC-100-2314-B-002-147-MY3, NTUH-103-082, NTUH-103-S-2467, NTUH-102-CGN03，NTUH102-S2097, NTUH 101-M1953, NTUH100-N1776, NHRI-PH-101-SP-09, and NHRI-PH-102-SP-09. The authors express their sincere gratitude to all participants of the NSARF (NSARF, The National Taiwan University Study Group on Acute Renal Failure).

\section{References}

[1] T. Z. Ali, I. Khan, W. Simpson et al., "Incidence and outcomes in acute kidney injury: a comprehensive population-based study," Journal of the American Society of Nephrology, vol. 18, no. 4, pp. 1292-1298, 2007.

[2] O. Liangos, R. Wald, J. W. O’Bell, L. Price, B. J. Pereira, and B. L. Jaber, "Epidemiology and outcomes of acute renal failure in hospitalized patients: a national survey," Clinical Journal of the American Society of Nephrology, vol. 1, no. 1, pp. 43-51, 2006.

[3] S. S. Waikar and W. C. Winkelmayer, "Chronic on acute renal failure: long-term implications of severe acute kidney injury," JAMA: Journal of the American Medical Association, vol. 302, no. 11, pp. 1227-1229, 2009.

[4] S. G. Coca, S. Singanamala, and C. R. Parikh, "Chronic kidney disease after acute kidney injury: a systematic review and metaanalysis," Kidney International, vol. 81, no. 5, pp. 442-448, 2012.

[5] S. G. Coca, B. Yusuf, M. G. Shlipak, A. X. Garg, and C. R. Parikh, "Long-term risk of mortality and other adverse outcomes after acute kidney injury: a systematic review and meta-analysis," American Journal of Kidney Diseases, vol. 53, no. 6, pp. 961-973, 2009.

[6] C. Mammen, A. Al Abbas, P. Skippen et al., "Long-term risk of CKD in children surviving episodes of acute kidney injury in the intensive care unit: a prospective cohort study," The
American Journal of Kidney Diseases, vol. 59, no. 4, pp. 523-530, 2012.

[7] E. Macedo, D. M. T. Zanetta, and R. C. R. M. Abdulkader, "Long-term follow-up of patients after acute kidney injury: patterns of renal functional recovery," PLoS ONE, vol. 7, no. 5, Article ID e36388, 2012.

[8] C. Hsu, G. M. Chertow, C. E. McCulloch, D. Fan, J. D. Ordoñez, and A. S. Go, "Nonrecovery of kidney function and death after acute on chronic renal failure," Clinical Journal of the American Society of Nephrology, vol. 4, no. 5, pp. 891-898, 2009.

[9] L. J. Lo, A. S. Go, G. M. Chertow et al., "Dialysis-requiring acute renal failure increases the risk of progressive chronic kidney disease," Kidney International, vol. 76, no. 8, pp. 893-899, 2009.

[10] N. Pannu, M. James, B. Hemmelgarn, and S. Klarenbach, "Association between AKI, recovery of renal function, and longterm outcomes after hospital discharge," Clinical Journal of the American Society of Nephrology, vol. 8, no. 2, pp. 194-202, 2013.

[11] J. Jones, J. Holmen, J. de Graauw, A. Jovanovich, S. Thornton, and M. Chonchol, "Association of complete recovery from acute kidney injury with incident CKD stage 3 and all-cause mortality," American Journal of Kidney Diseases, vol. 60, no. 3, pp. 402-408, 2012.

[12] R. Wald, R. R. Quinn, J. Luo et al., "Chronic dialysis and death among survivors of acute kidney injury requiring dialysis," JAMA: Journal of the American Medical Association, vol. 302, no. 11, pp. 1179-1185, 2009.

[13] G. M. Welten, O. Schouten, M. Chonchol et al., "Temporary worsening of renal function after aortic surgery is associated with higher long-term mortality," American Journal of Kidney Diseases, vol. 50, no. 2, pp. 219-228, 2007.

[14] C. E. Hobson, S. Yavas, M. S. Segal et al., "Acute kidney injury is associated with increased long-term mortality after cardiothoracic surgery," Circulation, vol. 119, no. 18, pp. 2444-2453, 2009.

[15] V. C. Wu, T. M. Huang, C. F. Lai et al., "Acute-on-chronic kidney injury at hospital discharge is associated with long-term dialysis and mortality," Kidney International, vol. 80, no. 11, pp. 12221230, 2011.

[16] A. Ishani, J. L. Xue, J. Himmelfarb et al., "Acute kidney injury increases risk of ESRD among elderly," Journal of the American Society of Nephrology, vol. 20, no. 1, pp. 223-228, 2009.

[17] H. Schiffl and R. Fischer, "Five-year outcomes of severe acute kidney injury requiring renal replacement therapy," Nephrology Dialysis Transplantation, vol. 23, no. 7, pp. 2235-2241, 2008.

[18] H. Schiffl, "Renal recovery from acute tubular necrosis requiring renal replacement therapy: a prospective study in critically ill patients," Nephrology Dialysis Transplantation, vol. 21, no. 5, pp. 1248-1252, 2006.

[19] M. Gallagher, A. Cass, R. Bellomo et al., "Long-term survival and dialysis dependency following acute kidney injury in intensive care: extended follow-up of a randomized controlled trial," PLoS Medicine, vol. 11, no. 2, Article ID e1001601, 2014.

[20] J. C. Luo, H. B. Leu, K. W. Huang et al., "Incidence of bleeding from gastroduodenal ulcers in patients with end-stage renal disease receiving hemodialysis," Canadian Medical Association Journal, vol. 183, no. 18, pp. E1345-E1351, 2011.

[21] C. Chang, J. Lin, H. Chen, C. Kuo, W. Shau, and M. Lai, "Non-steroidal anti-inflammatory drugs and risk of lower gastrointestinal adverse events: a nationwide study in Taiwan," Gut, vol. 60, no. 10, pp. 1372-1378, 2011. 
[22] S. H. Cheng, C. C. Chen, and W. L. Chang, "Hospital response to a global budget program under universal health insurance in Taiwan," Health Policy, vol. 92, no. 2-3, pp. 158-164, 2009.

[23] V. C. Wu, C. H. Wu, T. M. Huang et al., "Long-term risk of coronary events after AKI," Journal of the American Society of Nephrology, vol. 25, no. 3, pp. 595-605, 2014.

[24] Y. Lin, W. Ko, T. Chu et al., "The 90-day mortality and the subsequent renal recovery in critically ill surgical patients requiring acute renal replacement therapy," The American Journal of Surgery, vol. 198, no. 3, pp. 325-332, 2009.

[25] M. E. Charlson, P. Pompei, K. A. Ales, and C. R. MacKenzie, "A new method of classifying prognostic comorbidity in longitudinal studies: development and validation," Journal of Chronic Diseases, vol. 40, no. 5, pp. 373-383, 1987.

[26] A. O. Molnar, S. G. Coca, P. J. Devereaux et al., "Statin use associates with a lower incidence of acute kidney injury after major elective surgery," Journal of the American Society of Nephrology, vol. 22, no. 5, pp. 939-946, 2011.

[27] WHO, "The ATC and DDD system," http://www.whocc.noatc_ ddd_index/.

[28] L. S. Chawla, D. L. Davison, E. Brasha-Mitchell et al., "Development and standardization of a furosemide stress test to predict the severity of acute kidney injury," Critical Care, vol. 17, no. 5, R207, 2013.

[29] C. Baigent, M. J. Landray, C. Reith et al., "The effects of lowering LDL cholesterol with simvastatin plus ezetimibe in patients with chronic kidney disease (Study of Heart and Renal Protection): a randomised placebo-controlled trial," The Lancet, vol. 377, no. 9784, pp. 2181-2192, 2011.

[30] Y. Chen, J. Lin, H. Yu et al., "Cardiopulmonary resuscitation with assisted extracorporeal life-support versus conventional cardiopulmonary resuscitation in adults with in-hospital cardiac arrest: an observational study and propensity analysis," The Lancet, vol. 372, no. 9638, pp. 554-561, 2008.

[31] Y. Chou, T. Huang, V. Wu et al., "Impact of timing of renal replacement therapy initiation on outcome of septic acute kidney injury," Critical Care, vol. 15, no. 3, article R134, 2011.

[32] Y. C. Lee, W. Y. Shau, C. H. Chang, S. T. Chen, M. S. Lin, and M. S. Lai, "Antidepressant use and the risk of upper gastrointestinal bleeding in psychiatric patients: a nationwide cohort study in Taiwan," Journal of Clinical Psychopharmacology, vol. 32, no. 4, pp. 518-524, 2012.

[33] T. Huang, V. Wu, G. Young et al., "Preoperative proteinuria predicts adverse renal outcomes after coronary artery bypass grafting," Journal of the American Society of Nephrology, vol. 22, no. 1, pp. 156-163, 2011.

[34] V. C. Wu, W. J. Ko, H. W. Chang et al., "Early renal replacement therapy in patients with postoperative acute liver failure associated with acute renal failure: effect on postoperative outcomes," Journal of the American College of Surgeons, vol. 205, no. 2, pp. 266-276, 2007.

[35] C. C. Shiao, V. C. Wu, W. Li et al., "Late initiation of renal replacement therapy is associated with worse outcomes in acute kidney injury after major abdominal surgery," Critical Care, vol. 13, no. 5, article R171, 2009.

[36] W. J. Wang, C. T. Chao, Y. C. Huang, C. Y. Wang, C. H. Chang, and T. M. Huang, "The impact of acute kidney injury with temporary dialysis on the risk of fracture," Journal of Bone and Mineral Research, vol. 29, no. 3, pp. 676-684, 2014.

[37] V. C. Wu, T. M. Huang, P. Wu et al., "Preoperative proteinuria is associated with long-term progression to chronic dialysis and mortality after coronary artery bypass grafting surgery," PLoS ONE, vol. 7, no. 1, Article ID e27687, 2012.

[38] Y. C. Huang, S. J. Huang, and W. J. Ko, "Survey of do-notresuscitate orders in surgical intensive care units," Journal of the Formosan Medical Association, vol. 109, no. 3, pp. 201-208, 2010.

[39] A. Bihorac, S. Yavas, S. Subbiah et al., "Long-term risk of mortality and acute kidney injury during hospitalization after major surgery," Annals of Surgery, vol. 249, no. 5, pp. 851-858, 2009.

[40] R. L. Mehta, M. T. Pascual, S. Soroko et al., "Spectrum of acute renal failure in the intensive care unit: the PICARD experience," Kidney International, vol. 66, no. 4, pp. 1613-1621, 2004.

[41] D. P. Basile, D. Donohoe, K. Roethe, and J. L. Osborn, "Renal ischemic injury results in permanent damage to peritubular capillaries and influences long-term function," American Journal of Physiology-Renal Physiology, vol. 281, no. 5, pp. F887F899, 2001.

[42] M. Hörbelt, S. Lee, H. E. Mang et al., "Acute and chronic microvascular alterations in a mouse model of ischemic acute kidney injury," The American Journal of Physiology-Renal Physiology, vol. 293, no. 3, pp. F688-F695, 2007.

[43] R. A. Zager, A. C. M. Johnson, and K. Becker, "Acute unilateral ischemic renal injury induces progressive renal inflammation, lipid accumulation, histone modification, and "end-stage" kidney disease," American Journal of Physiology-Renal Physiology, vol. 301, no. 6, pp. F1334-F1345, 2011.

[44] C. T. Chao, V. C. Wu, C. Lai et al., "Advanced age affects the outcome-predictive power of RIFLE classification in geriatric patients with acute kidney injury," Kidney International, vol. 82, no. 8, pp. 920-927, 2012.

[45] R. Wald, S. Z. Shariff, N. K. Adhikari et al., "The association between renal replacement therapy modality and long-term outcomes among critically ill adults with acute kidney injury: a retrospective cohort study," Critical Care Medicine, vol. 42, no. 4, pp. 868-877, 2014.

[46] “2011 USRDS Annual Data Report,” 2011, http://www.usrds .org/2011/view/v2_12.asp.

[47] J. Bouchard, S. B. Soroko, G. M. Chertow et al., "Fluid accumulation, survival and recovery of kidney function in critically ill patients with acute kidney injury," Kidney International, vol. 76, no. 4, pp. 422-427, 2009.

[48] V. C. Wu, C. K. Wu, Y. C. Chang, G. H. Young, S. C. Chen, and W. S. Yang, "Association of the variations in the HSD3beta gene with primary aldosteronism," Journal of Hypertension, vol. 31, no. 7, pp. 1396-1405, 2013.

[49] F. Liaño, C. Felipe, M. T. Tenorio et al., "Long-term outcome of acute tubular necrosis: a contribution to its natural history," Kidney International, vol. 71, no. 7, pp. 679-686, 2007.

[50] E. D. Siew, J. F. Peterson, S. K. Eden et al., "Outpatient nephrology referral rates after acute kidney injury," Journal of the American Society of Nephrology, vol. 23, no. 2, pp. 305-312, 2012.

[51] Z. Harel, R. Wald, J. M. Bargman et al., "Nephrologist followup improves all-cause mortality of severe acute kidney injury survivors," Kidney International, vol. 83, no. 5, pp. 901-908, 2013.

[52] S. S. Waikar, R. Wald, G. M. Chertow et al., "Validity of international classification of diseases, ninth revision? clinical modification codes for acute renal failure," Journal of the American Society of Nephrology, vol. 17, no. 6, pp. 1688-1694, 2006. 


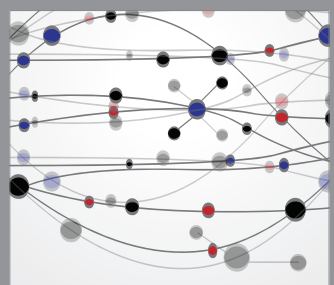

The Scientific World Journal
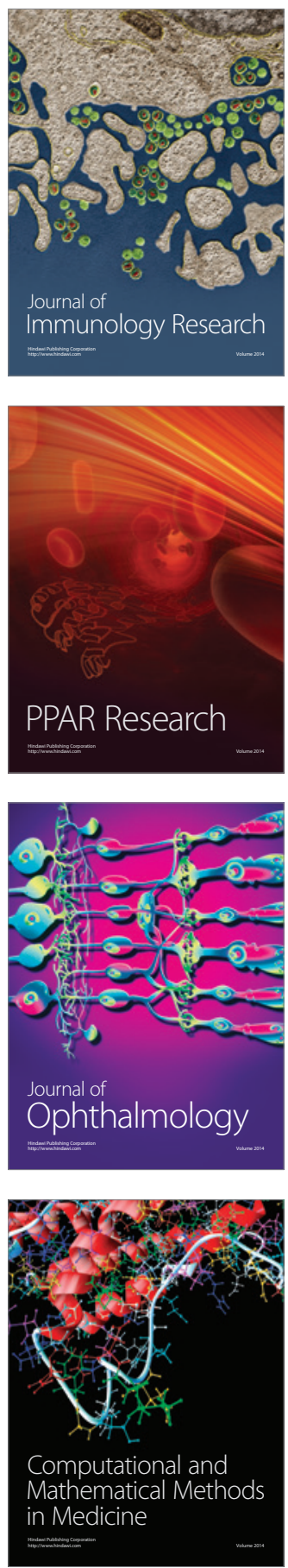

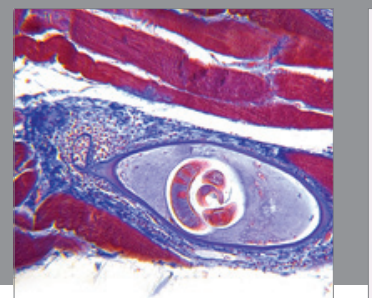

Gastroenterology

Research and Practice
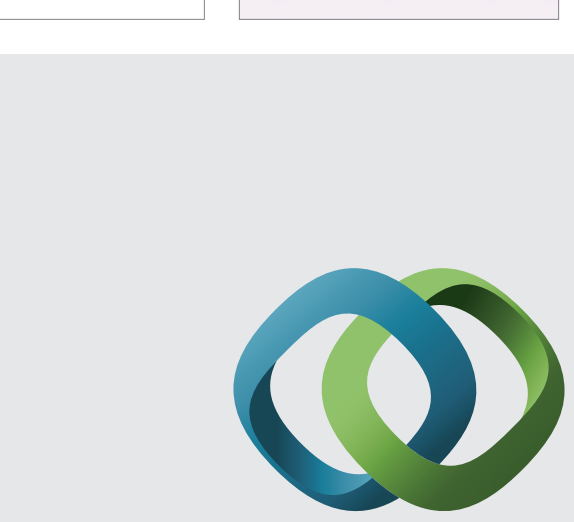

\section{Hindawi}

Submit your manuscripts at

http://www.hindawi.com
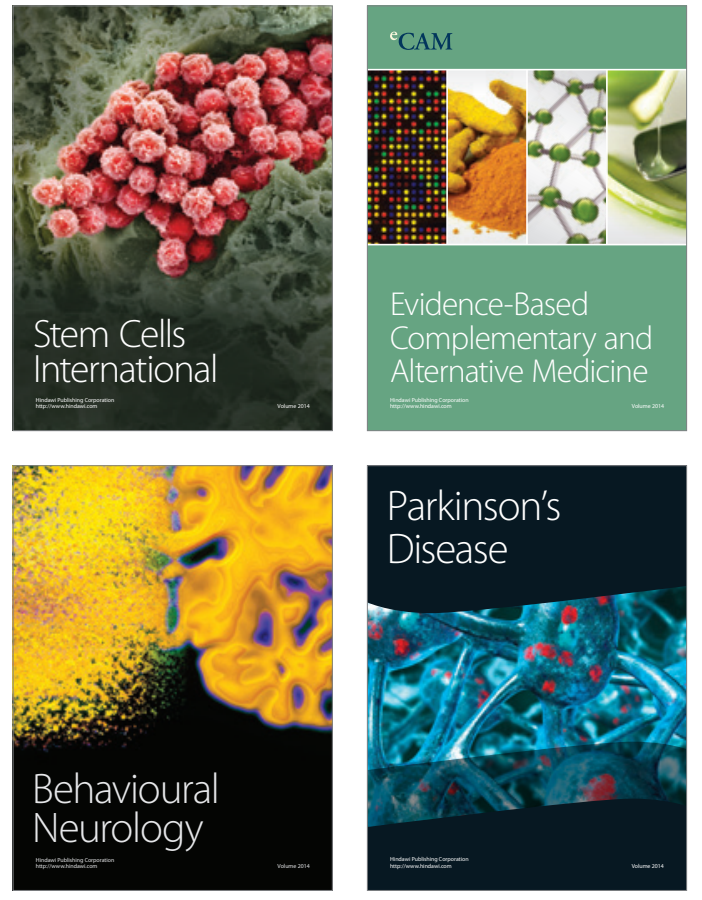
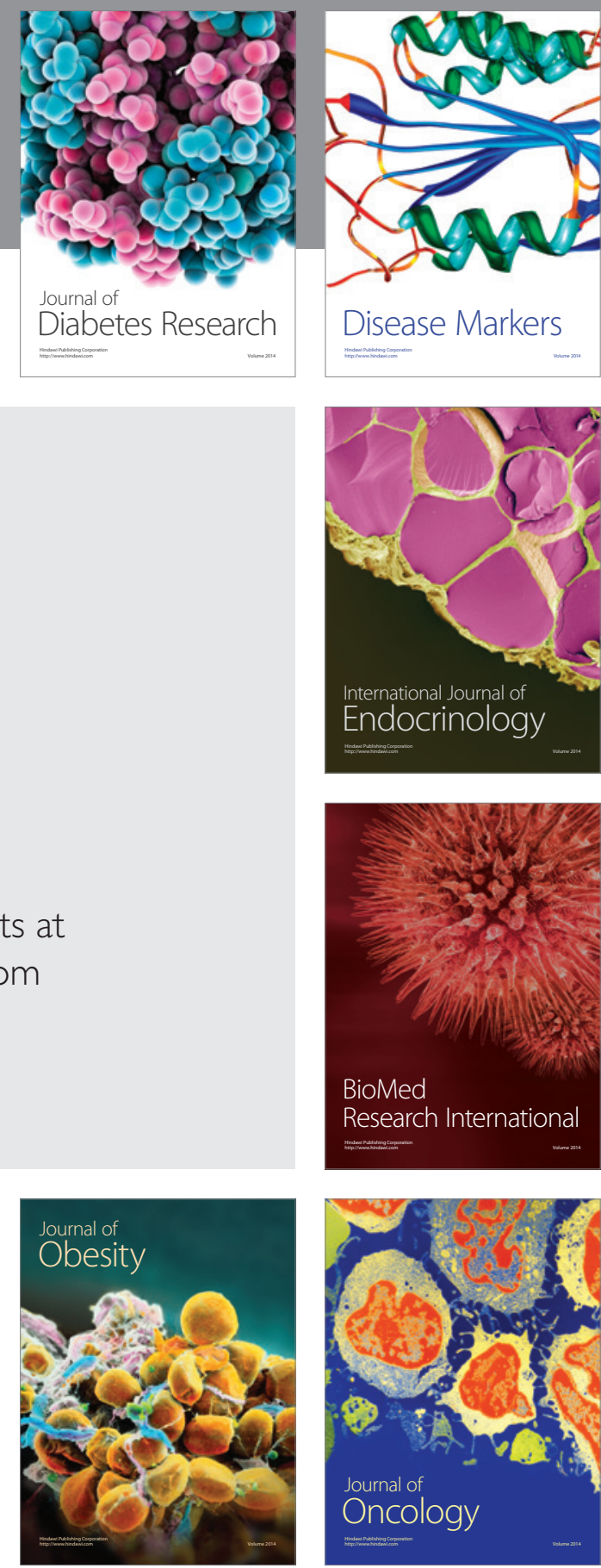

Disease Markers
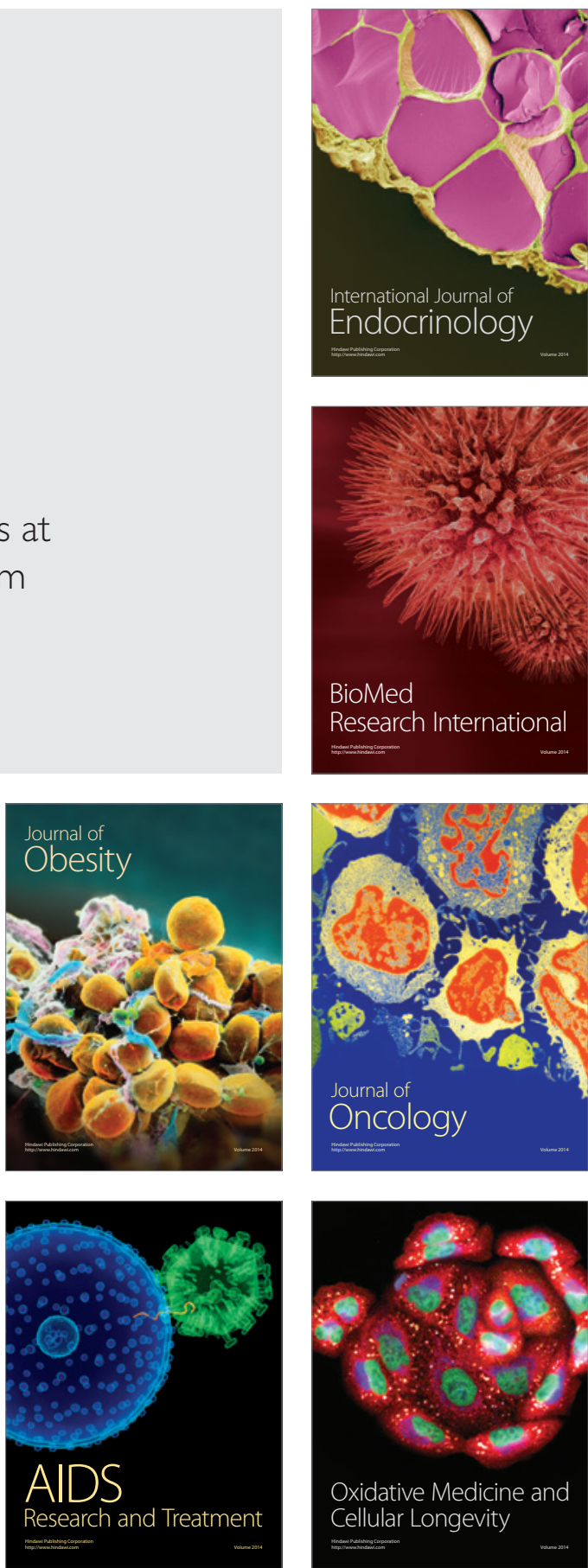